\title{
Intensity limitations of a gas cell for stopping, storing and guiding of
}

\section{radioactive ions}

\author{
Mark Huyse ${ }^{1,2, *}$, Marius Facina ${ }^{1}$, Yuri Kudryavtsev ${ }^{1}$, Piet Van Duppen ${ }^{1}$ and the \\ ISOLDE collaboration ${ }^{2}$ \\ 1 Instituut voor Kern-en Stralingsfysica, University of Leuven, Celestijnenlaan \\ 200D, B-3001 Leuven, Belgium \\ 2 CERN, CH-1211 Geneva, Switzerland
}

\begin{abstract}
The possibility to use a gas cell filled by noble gas (He or Ar) for thermalizing, storing and transporting radioactive ions is explored by studying experimentally ion - electron recombination of stable Ni, resonantly ionized by laser light. Combined with a literature study on ionization chambers, especially developed for high-intensity applications, conclusions are drawn on the maximum intensity of the incoming ion beam. A practical limit is encountered when the space-charge induced voltage fully counteract the applied voltage on the electrodes collecting the electrons.
\end{abstract}

July 14, 2001

PACS: 29.25; 29.40.Cs; 32.80.Fb; 41.85.Ar;

Keywords: Gas cell; Ion-electron recombination; Laser resonance ionization; Radioactive ion beams; Ionization chambers

* Corresponding author. Tel:+32-16-327272; fax:+32-16-327985

E-mail address: mark.huyse@fys.kuleuven.ac.be

\section{Introduction}

Chambers, filled with noble gasses such as helium and argon, are good devices to store ions of most of the chemical elements. The high ionization potential of the noble gas atoms prevents charge-exchange processes between the ions of interest and the buffer gas atoms resulting in long ion survival times. It is also possible to use high-density (around normal pressures) gasses for stopping energetic particles. Combining these two aspects does make gas cells an attractive approach to slow down radioactive ions after their production and transport them out of the gas cell to an on-line mass separator.

This transport can be through a gas flow inside the gas cell and a supersonic expansion at the exit hole of the cell. Depending on the dimensions of the cell and on the conductance of the exit hole, transport times will ranges from ms to several seconds. Such gas cells are presently in use at different accelerator laboratories and the approach is called the IGISOL-technique (Ion Guide Isotope Separation On Line). The dimensions of the cell are rather limited, as is the obtained efficiency due to the neutralization of the primary ions. For a recent overview see [1]. Re-ionization of the atoms of interest can be done resonantly by the use of laser light as done with the Leuven laser ion source (see [2] and references therein). 
A second and faster way to transport the ions is by using electrical fields inside the gas cell. This set-up will be called the drift gas cell. This type of gas cell is quite similar to ionization chambers used for detecting ionizing radiation. The total charge liberated by the radiation entering the gas cell (typical at 1 atmosphere and all kinds of gasses: He, Ar, $\mathrm{H}_{2}, \mathrm{~N}_{2}$, air) is collected on two electrodes (see figure 1). In order to have a good intensity and deposited-energy determination, no recombination of the positive and negative charges should occur.

Some methods developed to characterize and minimize recombination losses in gas cells will be reviewed here. Use will be made from recent experiments with the Leuven laser ion source and from the literature published on ionization chambers. Then, the primary conditions will be described to adapt a possible drift gas cell for the production of intense beams of radioactive ions. Where possible, the obtained results will be confronted with the experimental observations from ionization chambers, especially developed for highintensity applications, and from the laser ion source. Finally, a number of conclusions will be drawn on the applicability range of the drift gas cell.

\section{Recombination losses}

In ionization chambers one is interested in collecting all charges created by the ionizing radiation. In the application for slowing down and transporting radioactive ions one is in fact only interested in the particular radioactive ion. All other ion - electron pairs, created in the slowing down process, are rather a nuisance but it looks difficult to discriminate the two kind of ions and therefore one has also to take into account all created charges. Different chemical processes do take place in a gas cell influencing the fate of ions and atoms. For instance fast chemistry can take place between trace ions or atoms with impurities in the buffer gas or with the buffer gas atoms themselves (for a recent discussion see [2]). The most important loss factor of ions in the gas cell will be neutralization and the most important neutralization mechanism is the three-body ionelectron recombination. Taking the case where ion-electron $\left(\mathrm{X}^{+}-\mathrm{e}^{-}\right)$pairs are created in a gas cell filled with helium, the rate at which the three-body ion-electron recombination, $\mathrm{X}^{+}+\mathrm{e}^{-}+\mathrm{He}=>\mathrm{X}^{*}+\mathrm{He}$, is proceeding depends on $\alpha$, the recombination coefficient $\left(\mathrm{cm}^{3} \cdot \mathrm{s}^{-1}\right)$ and on $\mathrm{Q}$, the number of ion/e pairs created $\left(\mathrm{cm}^{-3} \cdot \mathrm{s}^{-1}\right)$. We will call $\mathrm{Q}$ the ionizing rate. As most of the ions will be buffer gas ions, the recombination process will be dominated by the recombination coefficient of the buffer gas itself. If there is a constant ionizing rate $\mathrm{Q}$, such as when a beam of ionizing radiation, having a constant intensity, passes through the gas cell, the time in which the three-body ion-electron recombination reaches the equilibrium ion-electron density can be determined as follows:

$$
t=\frac{1}{\sqrt{Q \alpha}}(\mathrm{s})
$$

In saturation, the density $\mathrm{n}$ is then:

$$
\mathbf{n}=\sqrt{\mathbf{Q} / \boldsymbol{\alpha}}\left(\mathrm{cm}^{-3}\right)
$$

For a helium ion in 0.5 atmosphere helium, the recombination coefficient $\alpha$ amounts to $1.6710^{-7} \mathrm{~cm}^{3} \cdot \mathrm{s}^{-1}[3]$. Taking an ionizing rate of $10^{10}$ ion-electron pairs $/ \mathrm{cm}^{3} \mathrm{~s}$ leads in 
helium to a saturation time of $24 \mathrm{~ms}$. It is thus clear that the ionizing rate $\mathrm{Q}$, the recombination coefficient $\alpha$ and the residence time of the ions and electrons in the gas will define recombination losses.

When using ionization chambers for Minimum Ionizing Particles (for a discussion see [4] and [5]), the number of ion-e pairs created per $\mathrm{cm}$ in 1 atmosphere $\mathrm{He}$ is 7.8. The mean ionization energy $\mathrm{W}_{\mathrm{i}}$ to create one ion-electron pair was taken to be $41 \mathrm{eV}$ (see table 1). In argon, due to the higher stopping efficiency and the lower mean ionization energy (26 $\mathrm{eV}$ ), this amounts to 94 pairs per $\mathrm{cm}$. The energy of the emitted electron varies between some $\mathrm{eV}$ up to several $\mathrm{keV}$ (called $\delta$ electrons): $80 \%$ are low-energetic $(<45 \mathrm{eV})$ against $0.2 \%$ high-energetic $(>3 \mathrm{keV})$. This means that some of these electrons can create more ion - electron pairs and therefore the multiplicity of ion-e pairs per interaction lies higher than 1 [5]. Furthermore these high-energy electrons will result through their energy loss in a much bigger pulse height, determining mainly the observed track diameter ( typically $100 \mu \mathrm{m})$. Taking this diameter, which is for most of the ion-electron pairs an upper limit, the ion density in the track is typically $10^{5}$ ion-e pairs $/ \mathrm{cm}^{3}$ for $\mathrm{He}\left(10^{6}\right.$ ion-e pairs $/ \mathrm{cm}^{3}$ for Ar). In contrast, the density of tracks of highly-ionizing particles is much higher. Highly-ionizing particles, discussed in the context of ionization chambers, are typically alpha particles and fission fragments. The beams of radioactive ions, envisaged to be stopped in a drift gas cell, range from He up to the superheavy elements.

In order to study neutralization in a gas cell, a controlled amount of ${ }^{58} \mathrm{Ni}$ ions, delivered by the CYCLONE cyclotron of Louvain-la-Neuve, was injected in the Leuven laser ion source [2]. Figure 2 gives a schematic overview of the experimental set-up. Starting at $185 \mathrm{MeV}$ and by proper energy degrading, a $30 \mathrm{MeV}^{58} \mathrm{Ni}$ beam enters the gas cell and is stopped in the middle when 0.5 atmosphere Ar is used. The beam spot has a diameter of about $6 \mathrm{~mm}$ and the longitudinal straggling spreads the stopped ions over $5 \mathrm{~mm}$ in the $\mathrm{y}$ direction (see figure 2). In figure 3, a number of time profiles of mass-separated beams after a $50 \mathrm{~ms}$ cyclotron pulse are given. Ions of ${ }^{40} \mathrm{Ar}$ are mainly flowing out of the gas cell during the beam impact indicating that the Ar buffer gas near the exit hole is directly ionized, probably by high-energetic photons and/or electrons. Ions of ${ }^{58} \mathrm{Ni}$, surviving the thermalization and transport in the gas cell, reach their maximum around $0.5 \mathrm{~s}$ after the impact (see ${ }^{58} \mathrm{Ni}$ off-resonance in fig. 3). This is in accordance with gas flow calculations on the used gas cell. If resonant laser light is used to re-ionize the neutralized ${ }^{58} \mathrm{Ni}$ beam particles, the ${ }^{58} \mathrm{Ni}$ signal has the same time profile but is some two orders of magnitude more intense (see ${ }^{58} \mathrm{Ni}$ on-resonance in fig. 3). The saw-tooth pattern is due to the $20 \mathrm{~Hz}$ laser pulse repetition rate and the fact that new neutral $\mathrm{Ni}$ atoms do enter the laser interaction zone (the cross section of the laser beam is $\sim 0.12 \mathrm{~cm}^{2}$ ). The time profiles in figure 3 were taken with a $\mathrm{Ni}$ beam of $1.5 \mathrm{pnA}$ (particle nano-Ampere). In figure 4, the mass-separated ${ }^{58} \mathrm{Ni}$ current is given as a function of the ${ }^{58} \mathrm{Ni}$ cyclotron beam current. A DC beam is applied here. The percentage of Ni ions, stopped in the middle of the gas cell, surviving the transport towards the exit hole in their ionic form is $0.5 \%$ when the impinging beam is less than $0.1 \mathrm{ppA}$. This decreases further to $0.033 \%$ when the beam amounts to $1 \mathrm{pnA}$ (the given efficiencies do take into account the transmission through the SPIG and through the mass separator, measured to be 60\%). This means that more than $99 \%$ of the original $\mathrm{Ni}$ ions are immediately neutralized. By applying resonant laser light some $10 \%$ can be re-ionized, at least when the primary beam is below 1 pnA. Above this value, saturation in the extracted current is observed. A number of 
conclusions can be drawn from the observations presented in the figures 3 and 4 . In figure 3, the Ar signal is only one order of magnitude larger than the $\mathrm{Ni}$ (off resonance) signal. But by stopping one $30 \mathrm{MeV}{ }^{58} \mathrm{Ni}$ ion in 0.5 atmosphere Ar a total of $30 \times 10^{6} / 26$ $1.1 \times 10^{6} \mathrm{Ar}^{+}$ion -electron pairs are created. This means that the $\mathrm{Ar}$ ions are even more efficiently neutralized than the Ni ions. Secondly, due to this strong neutralization, the effect of the high density of ion-electron pairs in the beam interaction zone diminishes fast. This can be seen from the strong Ni on - resonance signal in figure 3 and the constant efficiency of the laser re-ionization. Only at cyclotron intensities above $1 \mathrm{pnA}$ also recombination of the laser ions becomes important (see fig. 4). A rough calculation of the ion - electron density points to recombination effects in this set-up from $10^{8}$ ion electron pairs per $\mathrm{cm}^{3}$ on. There is another evidence for the previous statement: the dip in the $\mathrm{Ni}$ on - resonance time profile in figure 3 at the time of the beam impact points to a recombination process near the exit hole which is related to the high density of Ar ions at that instant. All this indicates that the local and momentary ion - electron density is crucial in determining the recombination rate.

Another way to study recombination in a pencil-like beam has recently been developed by using resonant laser ionization on trace elements released from a filament. In [2] such studies are presented: two collinear laser beams (cross section $\sim 12 \mathrm{~mm}^{2}$ ) resonantly ionize $\mathrm{Ni}$ atoms along their path in the gas cell, $5 \mathrm{~cm}$ long. In fact, the used set-up is very similar to the one discussed above but now the ${ }^{58} \mathrm{Ni}$ is not introduced by a cyclotron beam but by heating a Ni filament, evaporating neutral $\mathrm{Ni}$ atoms (see figure 2). The time profile of the ions coming out after one shot of the lasers is then recorded. By varying the temperature of the Ni filament and thus the concentration of $\mathrm{Ni}$ atoms, the intensity of the signal can be changed. No electrical fields are applied in the gas cell. Changing the ion - electron concentration in the laser interaction zone from $1 \times 10^{3}$ per $\mathrm{cm}^{3}$ to $4 \times 10^{4}$ per $\mathrm{cm}^{3}$ does not change the time profile (see figure 18 in [2]). But at an ion-electron density of $10^{7}$ per $\mathrm{cm}^{3}$ the signal drops indicating that a $\mathrm{Ni}$ ion has a considerable chance to recombine with an electron from another ion-electron pair. Such process is called volume recombination (see below) and is by definition rate-dependent. A proof for this can be found in the drop in intensity of potassium ions (see figure 18 and the related discussion in [2]). Potassium is constantly released from all parts of the gas cell and thermo-ionized by the Ni filament. A constant time profile is expected but at the densities where Ni ions start to recombine, thus during the production period of laser ions and electrons, the current of potassium is also reduced.

These two studies with the laser ion source indicate that even at time scales around $5 \mathrm{~ms}$, recombination losses start to be important once the ion-electron density is above $10^{7}-$ $10^{8} \mathrm{~cm}^{-3}$. Such densities are easily reached when the buffer gas is used to slow down energetic ions.

As an example for the further discussion, a beam of $\mathrm{Rh}$ ions will be considered. Using ion range calculations [6], now in 1 atmosphere of $\mathrm{He}$, a $\mathrm{Rh}$ ion will make $3.8 \times 10^{5}$ ion -e pairs per $\mathrm{cm}$ along its track when the energy of the beam is around the Bragg peak $(\sim 145$ $\mathrm{MeV})$. This decreases to $1.5 \times 10^{5}$ ion - e pairs per $\mathrm{cm}$ at $10 \mathrm{MeV}$ and to $4 \times 10^{4}$ ion - e pairs per $\mathrm{cm}$ at $10 \mathrm{keV}$ (here we take electronic and nuclear stopping). Taking some typically value of $8 \times 10^{4}$ ion - electron pairs per $\mathrm{cm}$ leads to a density along the track of one $\mathrm{Rh}$ ion of $8 \times 10^{8}$ ion-e pairs $/ \mathrm{cm}^{3}$. 
It is clear from these numbers and from the observations with stable Ni beams, that in order to collect all charges created by the passage of an intense ionizing beam, the threebody ion-electron recombination process should be stopped. The way out is to separate with an electrical field immediately the positive ions from the electrons and this is exactly what has been done for almost a century in ionization chambers.

Two types of recombination are considered in such a chamber: columnar or initial recombination where the ion recombines with an electron from the same track and volume or general recombination where the ion recombines with an electron from another track.

\section{$\underline{2.1 \text { Columnar (initial) recombination }}$}

A simple estimation of the field needed to separate the electrons from the ions is given in [7]. The average separation $d_{i}$ between the positive ions having a density $n_{i}$ is roughly:

$$
\mathbf{d}_{\mathbf{i}} \approx\left(\mathbf{n}_{\mathbf{i}}\right)^{-1 / 3}
$$

This is also the average separation between electrons and thus the Coulomb force $\mathrm{F}_{\mathrm{C}}$ acting between an ion-electron pair is:

$$
\mathbf{F}_{\mathbf{C}} \approx \frac{1}{4 \pi \varepsilon} \mathbf{e}^{2}\left(\mathbf{n}_{\mathbf{i}}\right)^{2 / 3}
$$

with $\varepsilon$ the di-electric constant of the gas ( $\boldsymbol{\varepsilon} \cong \boldsymbol{\varepsilon}_{0}$, the di-electric constant for vacuum) and e the charge unit.

The field needed to produce an equal and opposite force is then:

$$
\frac{\mathbf{V}_{\mathbf{a p p l}}}{\mathbf{d}} \approx \frac{1}{4 \pi \varepsilon_{0}} \mathbf{e}\left(\mathbf{n}_{\mathbf{i}}\right)^{2 / 3}
$$

Table 2 gives an estimation of the field strengths needed. Even at high ion - electron densities, the field strengths required are moderate. But this only gives a static description of the problem and time-dependent considerations should be implemented (see below).

Columnar recombination is in first order not rate-dependent. For highly-ionizing particles the saturation voltage, see figure 1, from where on all charges are collected and thus the columnar recombination losses are negligible, will be element- and energydependent. This will lead to a characteristic I - V plot and this principle is presently used in modern dosimetry detectors called recombination chambers [8]. Columnar recombination has been studied in great detail and methods have been developed to extract out of the I-V characteristics the difference between columnar and volume recombination [7]. Not only the field strength in the chamber is of importance but also the angle between the path of the ionizing radiation and the direction of the electrical field [9].

\subsection{Volume (general) recombination}

The ion cloud and the electron cloud from a single track move under the influence of an electrical field in opposite directions. When the ionizing rate is quite high, recombination can take place between ions and electrons from different tracks. This process is called volume or general recombination and is rate-dependent. The losses will depend on the 
time scale of the recombination, see equation $\{1\}$, and on the time spent by the ions and electrons before being collected on the electrodes.

The velocity of positive ions in a gas under the influence of a static electrical field $\mathrm{V} / \mathrm{d}$ is given as:

$$
\mathbf{v}_{+}=\mu_{+} \frac{\mathbf{V}}{\mathbf{d}}=\mu_{+} \mathbf{E}
$$

with $\mu_{+}$the mobility. The mobility depends on the temperature and pressure of the gas:

$$
\boldsymbol{\mu}_{+}=\boldsymbol{\mu}_{0} \frac{\mathbf{T}}{\mathbf{p} * 273}
$$

with the pressure $\mathrm{p}$ in atmosphere and the temperature $\mathrm{T}$ in $\mathrm{K}$. This means that the velocity has an $\mathrm{E} / \mathrm{p}$ dependence, which is a consequence of the fact that the average energy of the ions is almost constant during its drift to the electrode. Only at very high fields this dependence changes to $(\mathrm{E} / \mathrm{p})^{1 / 2}$. The situation is quite different for electrons as will be discussed below. The reduced field strength in a gas is expressed as E/N (with $\mathrm{N}$ the gas density) and given in Townsend $\left(1\right.$ Townsend $\left.=10^{-17} \mathrm{~V} \cdot \mathrm{cm}^{2}\right)$. Table 1 gives the classical mean free path, average velocity, diffusion coefficients and mobility of ions in like gas under normal conditions [4]. Most other ions in helium as buffer gas, have mobility coefficients around $20 \mathrm{~cm}^{2} / \mathrm{V} . \mathrm{s}[10]$.

The mobility of electrons is not constant as their energy increases, in between collisions with gas molecules, under the influence of the electric field. Table 3 gives the electron drift velocities in helium as deduced from measurements presented in [11]. The mobility of electrons is several orders of magnitude larger than the mobility of positive ions. Of prime importance to keep this fast drift is to prevent negative ion formation as those ions move with the speed of positive ions and thus some 1000 times slower than electrons. Such negative ions can be formed when impurities are present in the gas and for this reason $\mathrm{O}_{2}$ and water impurities should be kept below the ppm level. This is technically feasible $[12,2]$.

According to Sharpe [13] and later also Colmenares [14] the recombination loss $\mathrm{f}$ in ionization chambers (parallel plate chambers) can be estimated as:

$$
\mathrm{f}=\frac{\mathbf{Q} \alpha \mathbf{d}^{2}}{6 \mathbf{v}_{+} \mathbf{v}_{-}}
$$

with $\mathrm{d}$ the distance between the plates, $\alpha$ the recombination coefficient $\left(\mathrm{cm}^{3} \cdot \mathrm{s}^{-1}\right)$ and $\mathrm{Q}$ the ionizing rate $\left(\mathrm{cm}^{-3} \cdot \mathrm{s}^{-1}\right)$. Using the values for ion and electron mobility from table 1 and 3 and an ion-electron recombination coefficient $\alpha$ of $3.2510^{-7} \mathrm{~cm}^{3} \cdot \mathrm{s}^{-1}$ [3], the losses can be calculated for three configurations in 1 atmosphere He and in function of the electrical field strength, see table 4 . The production of the ion - electron pairs is assumed to be homogeneous over the volume between the two electrodes.

Other forms of chambers (cylindrical or spherical) are also calculated in [13] and typically give a factor 2 to 8 more losses (due to field geometry).

Inspecting table 4 shows that for all configurations quite high ionizing rates can be accepted by the ionization chamber provided large field-strengths are used. However this brings us to another limiting factor namely space-charge effects resulting from the high density of positive ions. 


\section{Space-charge effects}

Sharpe [13] calculates the voltage induced by the slowly moving positive ions in the ionization chamber and thus screening the applied electrical field. The electrons are assumed to be collected immediately. For a parallel plate chamber the following equation is derived:

$$
\mathbf{V}_{\text {ind }}=\sqrt{\frac{\mathbf{e Q}}{4 \boldsymbol{\varepsilon}_{0} \boldsymbol{\mu}_{+}}} \mathbf{d}^{2}
$$

When the net field at the anode is reduced to zero, the chamber is called to be spacecharge limited. Using the value for the He ion mobility from table 1 , the ionizing rate $\mathrm{Q}$ can be calculated where the space-charge induced voltage $V_{\text {ind }}$ equals the bias voltage $\mathrm{V}_{\text {appl }}$ for three values of $\mathrm{d}$ and in function of the electrical field strength, see table 5. By comparing table 4 and 5 it is clear that space-charge limitations are occurring already at lower rates than those where volume recombination losses become important. The effect of the space charges is that the net field that the ions do feel will decrease leading to a reduced velocity and thus a higher space charge. Furthermore the electron velocity will also be reduced and this will lead together with the reduced ion mobility to an increase in recombination, see equation $\{8\}$. The values from table 5 give thus only a rough estimate which field strengths should be used for a given ionizing rate in order to minimize recombination effects. Calculations combining the cumulative effects are in progress. Other forms of chambers (cylindrical or spherical) are also calculated in [13] and typically give limiting ionizing rates $\mathrm{Q}$ which are a factor of 10 lower (due to field geometry).

Ionization chambers are currently developed for precise beam monitoring of synchrotron radiation [15], [16] and of high-energetic ( $\mathrm{GeV} /$ nucleon) $p, d$ and $\alpha$ beams [17]. In the first case, $\mathrm{X}$-ray photon (around $20 \mathrm{keV}$ ) rates up to $10^{12}$ photons/s were directed to the chamber (1 atmosphere Ar) creating ionization currents up to the $0.1 \mathrm{~mA}$ level. High beam intensities could also be measured in the second case: up to $10^{11}$ protons per spill ( 1 spill every $1.5 \mathrm{~s}$ ). In figure 5 , some saturation field strengths are given in function of the ionizing rate $\mathrm{Q}$ for the three ionization chambers. The saturation field strength is the electrical field strength in which for a given incoming beam intensity the measured current saturates (see figure 1). This does not mean that the ionization chamber has no recombination losses anymore and different methods can be used to determine the saturation current [7]. However for the discussion presented here, the saturation voltage is deduced from inspecting the $\mathrm{I}-\mathrm{V}$ (current versus voltage) plots presented in the different articles. In all cases, the gas was argon but similar values for other gasses are also presented in [15]. The distance between the two parallel plates varied from 0.8 to $1.25 \mathrm{~cm}$, thus in the range of the $1-\mathrm{cm}$ arrangement presented in table 5 . All measurements do show a square-root dependence of $\mathrm{Q}$, the ionizing rate, as expected from equation $\{9\}$. In order to show this behavior, the calculation for different electrode distances of the induced voltage by space-charge effects given by equation $\{9\}$ and using the mobility coefficient for Ar is also given in figure 5. The measurements of Sugaya et al. for protons [17] coincides with those from Ahmed et al. for $17 \mathrm{keV}$ x-rays and qualitatively agree with $1 \mathrm{~cm}$ electrode gap calculation. The measurements of Sato et al. do deviate from the others. In contrast the beam here is extremely focussed to a spot size 
of $0.5 \times 0.6 \mathrm{~mm}^{2}$ in the middle of the gap between the electrodes $(\mathrm{d}=1.25 \mathrm{~cm})$ while in the other cases the beam is distributed uniformly over the gap, as also assumed in equation $\{9\}$. The last point measured by Sato et al. (at a field strength of $2000 \mathrm{~V} / \mathrm{cm}$ ) did not give full saturation yet for the given ionizing rate. In fact, the maximum applicable voltage of the chamber before breakdown limits this experimental point.

Palestini et al. [18] also discuss space charge effects in parallel plate ionization chambers and they demonstrate that in the very high intensity case a field-free region adjacent to the anode is created, expanding as the intensity of the ionizing beam increases.

\section{Plasma effects}

In ionization chambers one should in principle not consider plasma effects as the electrons are immediately collected and no neutral plasma can be created. However once the space-charge limit is reached the situation will be totally different and the field-free region as discussed by [18] will eventually expand over the whole cell: a weakly-ionized plasma $\left(\mathrm{n}_{\mathrm{i}} \sim \mathrm{n}_{\mathrm{e}}<<\mathrm{n}_{0}\right.$ with $\mathrm{n}_{\mathrm{i}}, \mathrm{n}_{\mathrm{e}}$ and $\mathrm{n}_{0}$ respectively the ion-, electron- and neutrals density) will be formed. Of course in such plasma the recombination rate is high and the gas cell cannot longer be used to store and transport efficiently the incoming ions. The ion and electron density will similarly evolve to a saturation value given by equation $\{2\}$ in a time given by equation $\{1\}$. It is difficult to estimate the electron temperature in the gas cell and it could well be that the plasma is not in thermal equilibrium, therefore it is not easy to fully characterize the plasmas observed in for instance the IGISOL gas cell. A consequence of plasma formation is that collective effects do emerge. An important observation is the occurrence of ambipolar diffusion where the ions and electrons do move with the same velocity $\left(v_{\text {electrons }} \sim v_{+}\right)$. A necessary condition is that the Debeye length of the plasma is of the same order or less then the dimension of the cell.

The Debeye length $\lambda_{\mathrm{D}}$ (in $\mathrm{mm}$ ) is given by the following expression [19]:

$$
\lambda_{\mathrm{D}}=69 \sqrt{\frac{\mathbf{T}_{\mathbf{e}}}{\mathbf{n}_{\mathbf{e}}}}
$$

with $\mathrm{T}_{\mathrm{e}}$ the electron temperature in ${ }^{\circ} \mathrm{K}$ and $\mathrm{n}_{\mathrm{e}}$ the electron density in $\mathrm{cm}^{-3}$. Assuming the electrons at room temperature and a density of $10^{8} \mathrm{~cm}^{-3}$, the Debeye length is already in the $0.1-\mathrm{mm}$ range.

A second phenomenon is that the plasma shields itself for external fields through the formation of a plasma sheath. The Debeye length is a measure for the penetration of external fields in the plasma. A necessary condition is that the number of charges in the plasma sheath region $N_{D}$ is much larger than 1 . This number is given by [19]:

$$
\mathbf{N}_{\mathbf{D}}=1380 \frac{\mathbf{T}_{\mathbf{e}}^{3 / 2}}{\mathbf{n}_{\mathbf{e}}^{1 / 2}}
$$

Taking the same conditions as just above $\mathrm{N}_{\mathrm{D}}$ lies in the range of 700 particles. Another condition states that the number of collisions with neutral atoms should be small. This condition can be quantified as follows [19]:

$$
\omega \tau>1 \quad \text { with } \omega \approx 9000 \sqrt{\mathbf{n}_{\mathbf{i}}} \text { and } \tau=\frac{\lambda}{\mathbf{v}_{\mathbf{a v}}}
$$


Using an ion - electron density of $10^{8}$ ions per $\mathrm{cm}^{3}$ and the values from table 1 for $\lambda$, the mean free path and $v_{\mathrm{av}}$, the average velocity, the product of $\omega$, the plasma oscillation frequency, and the mean time $\tau$ in between two collisions in the helium gas lies around 0.02 . Clearly this third condition is not fulfilled and one only can speak of a weaklyionized plasma.

The term "plasma effects" can be found in many publications on IGISOL set-ups [20]. The term is used to discuss the losses of ion-guide efficiency due to the intensity of the incoming primary beam and related to this, the rate of three-body recombination. Although some conflicting results can be found in the literature, Dendooven [20] concludes that in most measurements a drop in ion-guide efficiency is observed when the energy deposition in the ion guide stopping chamber leads to the creation of more than $10^{16}$ ion - electron pairs per second. However in the same survey article [20], evidence is presented where the drop in efficiency seems to be related to extraction problems near the exit of the gas cell. The IGISOL approach is based on the evacuation of important parts of the gas cell within time scales shorter than the time scale of the three-body recombination (see equation $\{1\}$ ). The obtained efficiency will be the result of a multiparameter balance involving the gas cell design, the beam properties, the purity of the gas and the extraction conditions. Therefore it is difficult to compare the results obtained at different laboratories or even within the same laboratory if the above-mentioned parameters are not controlled within a reproducible way. In any case the results of the studies with ion guide set-ups cannot directly been used for the drift gas cell as here the concept is completely different with the use of electrical fields to collect all created electrons.

\section{Application to the production of radioactive ion beams.}

The basic question will now be addressed if a big gas cell (from several $\mathrm{cm}^{3}$ to several liters) at moderate pressures ( 0.1 to 1 atmosphere He or Ar) can be used to slow down radioactive ions and keep them in their ionic state in order to transport them fast and efficient to a mass separator. It is clear from the considerations above that electrical fields should be firstly used to immediately collect the electrons in order to prevent columnar and volume recombination and secondly to guide the ions out of the cell. As described above, the number of ion-electron pairs (Q) created per $\mathrm{cm}^{3}$ and per $\mathrm{s}$ is the most important parameter. Table 6 gives this quantity for a number of specific applications for the production of radioactive ion beam.

- The first case describes the passage of a primary beam, used to produce radioactive nuclei in a heavy-ion fusion reaction, through the gas cell. The chamber will be immediately space-charge limited and a weak plasma will be the result. Recent experiments with the laser ion source at the LISOL facility [2] were conducted to investigate to what extent it was possible to use electrical fields inside the gas cell to collect all charges created by the passage of an ionizing laser beam through the cell (see figure 2 ). It was shown there that using a small voltage $(3 \mathrm{~V})$ on the grid, it was possible to effectively remove the laser-produced electrons and stop ion- electron recombination. Furthermore, by increasing the voltage above $30 \mathrm{~V}$, ions from deeper in the gas cell were collected on the grid while ions in between the grid and the exit 
hole were pushed out faster. Here we present an on-line measurement with the same set-up: ${ }^{58} \mathrm{Ni}$ atoms are again evaporated from a filament but now they are ionized by an impinging ${ }^{40} \mathrm{Ar}$ beam. From figure 6 it is clear that the electrical field does have an impact on the ions on their way out of the gas cell. But not all charges are collected: the weak plasma shields its interior from the external field as otherwise the hatched area in figure 6 would not be present. Indeed, from table 4, it is clear that even for a electrode gap distance of $1 \mathrm{~cm}$ a too high field strength is needed to get above the space-charge limitation when an ionizing rate of $2.610^{14}$ ion - electron pairs per $\mathrm{cm}^{3}$ and per $\mathrm{s}$ is entering the gas cell.

- Case 2 refers to the use of a gas cell for producing beams of fission products [20, 12]. Here only the fission products are entering the gas cell that is shielded from the primary proton beam. However even now, the ion-electron pair production is so high that the space-charge limit is reached. The conclusion is that also in this case it is extremely difficult to use electrical fields inside the gas cell. Especially when taking into account that due to the specificity of the application, the electrodes should lie some 2 to $3 \mathrm{~cm}$ apart and that a cylindrical geometry is needed.

- The third case comes from the work at Mainz [21] where resonant laser light is used to re-ionize the wanted nuclei. Electrical fields have been successfully used to collect all the ${ }^{242 \mathrm{~m}}$ Ar ions surviving neutralization during the thermalization in the gas cell. Indeed the ionizing rate is so low that space-charge effects can be ignored.

- Case 4 describes the possible application of a drift gas cell coupled to the SHIP velocity separator, the so-called SHIPTRAP project [22]. In heavy-ion fusion reactions, the produced isotopes can be in-flight very efficiently separated from the primary beam. At the focal plane a gas cell with dimensions adapted to the beam size can then be used to store and transport the radioactive ions. A typical distance between the electrodes would be $10 \mathrm{~cm}$ and according to table 5, a field strength above $10 \mathrm{~V} / \mathrm{cm}$ would be needed to overcome the space-charge limitations.

- Case 5 describes the parameters for a gas cell coupled to a heavy-ion fragment separator. G. Savard et al. are proposing such a gas cell for producing intense radioactive ion beams [23]. A heavy-ion beam of $400 \mathrm{MeV} / \mathrm{amu}$ impinges on a fragmentation production target after which the isotope of interest is separated in the fragment separator. Assuming a full suppression of the primary beam and of all unwanted reaction products such as isobaric contaminants, a typical beam further considered here could be $10^{8}{ }^{\mathrm{A}} \mathrm{Rh}$ atoms per s. By a careful ion-optical design of the fragment separator it should be possible to energy focus the relativistic heavy ions in order to bunch the range distribution of the fragments [24]. The kinetic energy of the slowed-down products entering the gas cell is expected to range between 0 to 20 $\mathrm{MeV} / \mathrm{u}$. In order to account for the differences in ion energies and straggling for the different ion paths in the gas cell a full calculation of the specific reaction is needed. However as we are only interested in orders of magnitude, for the purpose of simplicity a mono-energetic ${ }^{\mathrm{A}} \mathrm{Rh}$ beam at $650 \mathrm{MeV}$ will be considered. Such a 650 $\mathrm{MeV}$ beam would be stopped in 1 meter of $0.5 \mathrm{~atm} \mathrm{He}$. This means that one $\mathrm{Rh}$ 
projectile creates $650^{*} 10^{6} / 41=1.6^{*} 10^{7}$ ion-e pairs along its path. Taking a volume of $10^{5} \mathrm{~cm}^{3}$ and an intensity of $10^{8}$ atoms per s this gives an ionizing rate of $\mathrm{Q}=$ $1.6 * 10^{10}$ ion-e pairs $/ \mathrm{cm}^{3} \mathrm{~s}$. Also here the actual stopping process is simplified as the stopping power $\mathrm{dE} / \mathrm{dx}$ along the path depends on the energy. The dimensions of the cell are such that electrode distances will lie between 10 and $50 \mathrm{~cm}$ which, according to table 5, will imply extremely high field strengths in order to compensate spacecharge effects. A scaled-down cell is presently used at ATLAS and extraction fields up to $10 \mathrm{~V} / \mathrm{cm}$ were reached [23].

The general problem one is encountering is that in order to keep the one ion of interest, the particular ${ }^{\mathrm{A}} \mathrm{Rh}$ ion, all the ions from the ion-electron pairs created in the slowingdown process are also surviving. Until now we only considered space-charge effects disturbing the electrical field needed for collecting the electrons, but serious distortions could also be expected at the exit hole of the gas cell. Taking up again the example of the $10^{8} 650 \mathrm{MeV}{ }^{\mathrm{A}} \mathrm{Rh}$ beam, when no neutralization occurs a total current of $250 \mu \mathrm{A}$ is created. Extracting these intense currents by static or RF fields in the differential pumping zone where the transition from high gas pressures in the gas cell to high vacuum in the mass separator takes place will need special care. At Jyväskylä, where sometimes currents up to $20 \mu \mathrm{A}$ are extracted out of the IGISOL gas cell, transmission problems with the static skimmer electrode have been observed [20].

Maybe methods can be developed to collect the He ions on the electrodes without disturbing too much the trajectory of the heavier ions on their way out of the gas cell. The difference in mobility of $\mathrm{He}^{+}$ions in $\mathrm{He}$ and other ions in $\mathrm{He}$ (only a factor of two) seems to be quite low for this purpose.

Another possibility consists in re-ionizing the atoms of interest as done by resonant laser light at the LISOL set-up $[12,2]$. Losses during transport of the neutral atoms to the laser interaction zone, due to diffusion to the wall or to chemical interactions with impurity molecules or buffer gas atoms should be considered when designing the cell.

\section{Conclusions}

Starting from the century-old experience of gas-filled ionization chambers and recent measurements with laser ionization in a gas cell, the possibilities have been explored to use large gas cells for stopping, storing and guiding radioactive ions towards a mass separator in order to obtain intense beams of short-living nuclei. A practical limit is encountered for the maximum intensity of the beams of interest when the space-charge induced voltage fully counteract the voltage applied on the electrodes collecting the electrons. Such limit is also encountered in ionization chambers used for high-intensity beam monitoring. If no solution can be found for these problems, the applicability of the gas cell will be limited by the maximal field strength that can be applied, by the ionizing rate of the beam and by the maximum charge that can be extracted out of the cell. 


\section{References}

[1] J. Aysto, accepted for publication in Nuclear Physics A

[2] Y. Kudryavtsev et al., Nucl. Instr. and Meth. B179 (2001) 412

[3] A. Wayne Johnson, J. B. Gerardo, Phys. Rev. A7 (1973) 1339

[4] F. Sauli, "Principles of operation of multiwire proportional and drift chambers" in "Experimental Techniques in High-energy Nuclear and Particle Physics", World

Scientific, Ed. Thomas Ferbel (1991)

[5] H. Tolsma, Ph. D thesis (1996) http://www.nikhef.nl/ i94/thesis/node17.htm

[6], J. F. Ziegler, J. P. Biersack and U.Littmark,"The Stopping and Range of Ions in Solids", Pergamon Press, New York, 1985

[7] S. M. Mustafa and K. Mahesh, Nucl. Instr. and Meth. 150 (1978) 549 - 553

[8] M. Zielczynski et al., Nucl .Instr. Meth. A370 (1996) 563 - 567

[9] W. R. Kanne and J. A. Bearden, PR 50 (1936) 935 - 938

[10] H. W. Ellis, At. Nucl. Data Tables 31 (1984) 122

[11] P. Rice-Evans, "Spark, streamer, proportional and drift chambers", The Richelieu Press Limited, London (1974)

[12] Y. Kudryavtsev et al., Nucl. Instr. and Meth. B114 (1996) 350

[13] J. Sharpe, Nuclear Radiation detectors, Methuen \&Co., (1964)

[14] C. Colmenares, Nucl. Inst. and Meth. 114 (1974) 269 -275

[15] K. Sato et al., "The behavior of ionization chambers under the irradiation of highflux x-ray beam, SPRING8 annual report (1997), www.spring8.or.jp/ENGLISH/publication/ann_rep/

[16] S. N. Ahmed et al., Nucl. Instr. and Meth. A449 (2000) 248 - 253

[17] Y. Sugaya et al., Nucl. Instr. and Meth. A368 (1996) 635

[19] F. C. Chen, "Introduction to plasma physics", Plenum Press (1974)

[20] P. Dendooven, Nucl . Instr. and Meth. B126 (1997) 182 - 189

[21] H. Backe et al., Nucl. Instr. and Meth. B126 (1997) 406

[22] SHIPTRAP home page: http://www-aix.gsi.de/ shiptrap/index.html

[23] G. Savard et al., to be published in Nuclear Instruments and Methods

[24] H. Weick et al., ECT* workshop on "Physics with radioactive beams: key experiments and requirements", Trento, Italy, 27 Nov. - 1 Dec.

2000,http://www.ect.it/workshops/000112 


\section{Figure captions}

Figure 1

A schematic presentation of a gas-filled ionization chamber. A beam with an ionizing rate $\mathrm{Q}$ enters the gas cell and the created ions and electrons will be collected on the respective electrodes. At low electrode voltages, the recombination rate will be high. But eventually at the so-called saturation voltage, the extracted current will reach its full maximum.

\section{Figure 2}

The Leuven laser ion source. Stable Ni ions are brought in by a cyclotron beam while neutral $\mathrm{Ni}$ atoms can be evaporated from a filament. Re-ionization can resonantly been done by laser light. The ions leaving the cell are mass-analyzed by an isotope separator. A grid in the gas cell can be biased in order to study the effects of an electrical field.

\section{Figure 3}

A pulsed beam of ${ }^{58} \mathrm{Ni}$ (50 ms width) enters the Leuven laser ion source. The time behavior of the extracted $\mathrm{Ar}^{+}$and $\mathrm{Ni}^{+}$ions is recorded (later times means ions from deeper in the gas cell). The Ni signal can come from primary Ni ions surviving neutralization during their journey in the gas cell $\left({ }^{58} \mathrm{Ni}\right.$ off - resonance) or from re ionized laser ions $\left({ }^{58} \mathrm{Ni}\right.$ on - resonance).

\section{Figure 4}

The mass-separated ${ }^{58} \mathrm{Ni}$ current as a function of the incoming ${ }^{58} \mathrm{Ni}$ cyclotron beam current. The straight lines give the expected current for a $100 \%$ (full), $10 \%$ (dashed) and $1 \%$ (pointed) survival efficiency. The triangles give the obtained current of surviving Ni ions (off-resonance) and the circles the current after laser re-ionization (on-resonance).

\section{Figure 5}

For three different ionization chambers filled with argon as buffer gas (diamonds, circles and triangles refer respectively to the work of Sugaya et al. [17], Ahmed et al. [16] and Sato et al. [15]), the field strength needed to reach the plateau in the I-V plot is given as a function of $\mathrm{Q}$, the ionizing rate. Also given is the calculated field strength for a a number of electrode distances as a function of $Q$ using equation $\{9\}$ for $\operatorname{Ar}\left(\mu^{+}=1.7 \mathrm{~cm}^{2} / \mathrm{V} . \mathrm{s}\right)$.

The five cases, given in table 6 and discussed in the text, are also given; note that for case 4 and 5 helium is used as buffer gas and therefore the results from table 5 are used.

\section{Figure 6}

The influence of an electrical field for blocking and collecting the ions produced through the impact of a pulsed ${ }^{40} \mathrm{Ar}$ beam. The current at mass ${ }^{58} \mathrm{Ni}$ is measured with (thick line) and without the blocking field (thin line). The ${ }^{58} \mathrm{Ni}$ is evaporated as neutral atoms from a filament and is then ionized through the beam impact. 


\section{Table captions}

\section{Table 1}

The classical mean free path $\lambda$, average velocity $\mathrm{v}_{\mathrm{av}}$, mean energy to create an ion electron pair $\mathrm{W}_{\mathrm{i}}$, diffusion coefficient $\mathrm{D}^{+}$and mobility $\mu^{+}$of ions in like gas under normal conditions [4]

\section{Table 2}

Field strengths needed to produce a repulsive force counteracting the attractive Coulomb force between ions and electrons at a given density $n_{+}=n_{-}=n_{i}$.

\section{Table 3}

Electron drift velocities in helium as deduced from measurements presented in [11].

\section{Table 4}

Volume recombination losses in parallel plate ionisation chamber filled with 1 atmosphere helium $\left(\mu_{0}=10.2 \mathrm{~cm}^{2} /\right.$ V.s $)$ in function of the ionisation rate $\mathrm{Q}$, the applied electrical field $\mathrm{E}$ and the distance $\mathrm{d}$ between the plates.

\section{Table 5}

By using equation $\{9\}$ for He as gas $\left(\mu^{+}=10.2 \mathrm{~cm}^{2} /\right.$ V.s $)$ the ionizing rate $\mathrm{Q}$ is calculated for which the field strength induced by space-charge effects equals the applied field strength. Three different distances between the parallel electrode-plates are given.

\section{Table 6}

The number of ion-e pairs created per $\mathrm{cm}^{3}$ and per $\mathrm{s}$ in a gas cell by the passage of different beams. The dimensions of the gas cell have been adapted to the reactions. The p-induced fission relates to the work at Leuven [12] and Jyväskylä [20] where the primary beam is shielded from the gas cell with a thin foil. The ${ }^{242 \mathrm{~m}} \mathrm{Am}$ refers to the work of Mainz [21], also here the primary beam is not entering the gas cell. The example of the $65 \mathrm{MeV}$ Po beam is illustrative for radioactive beams produced in 


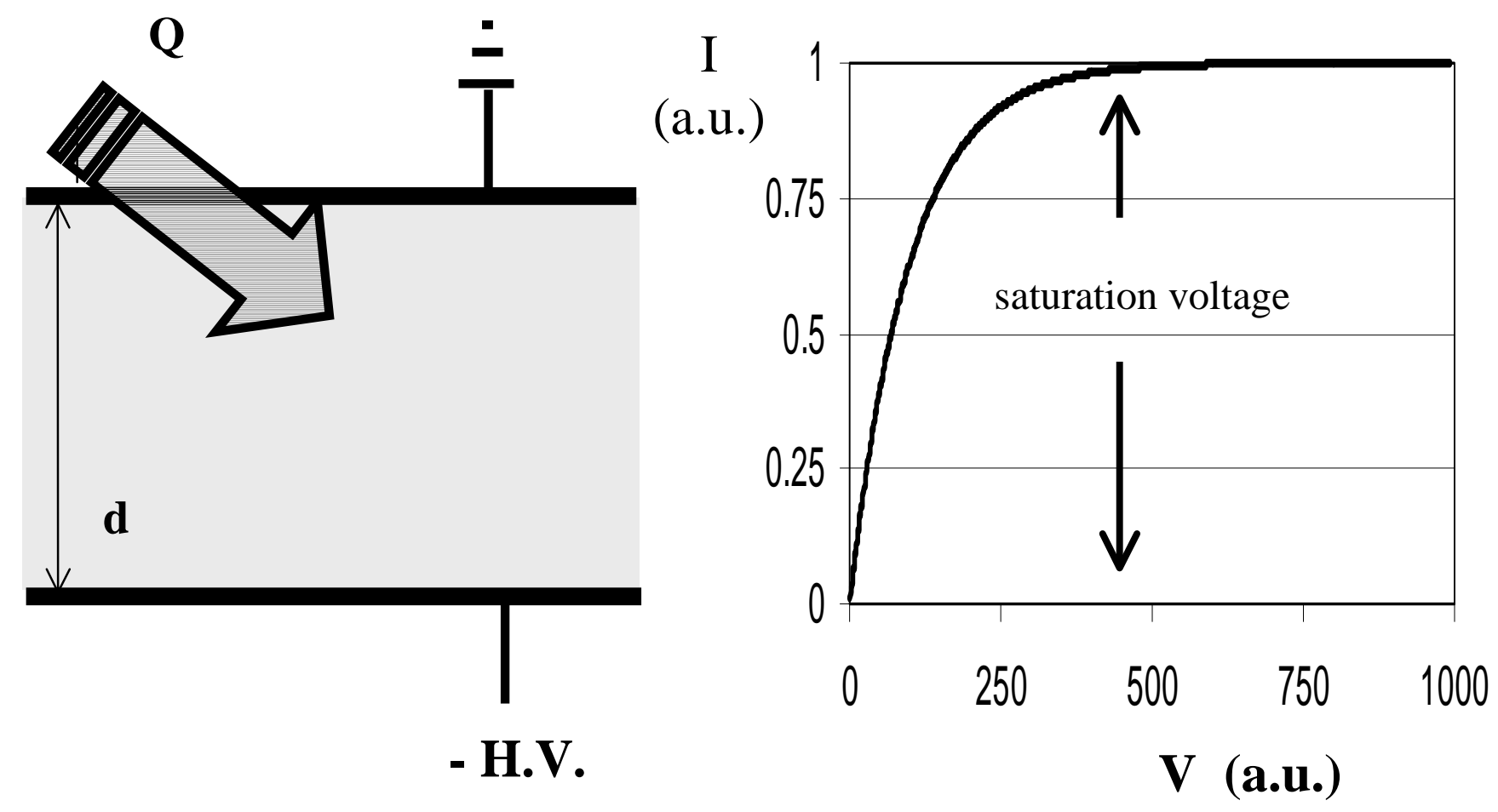

Figure 1 

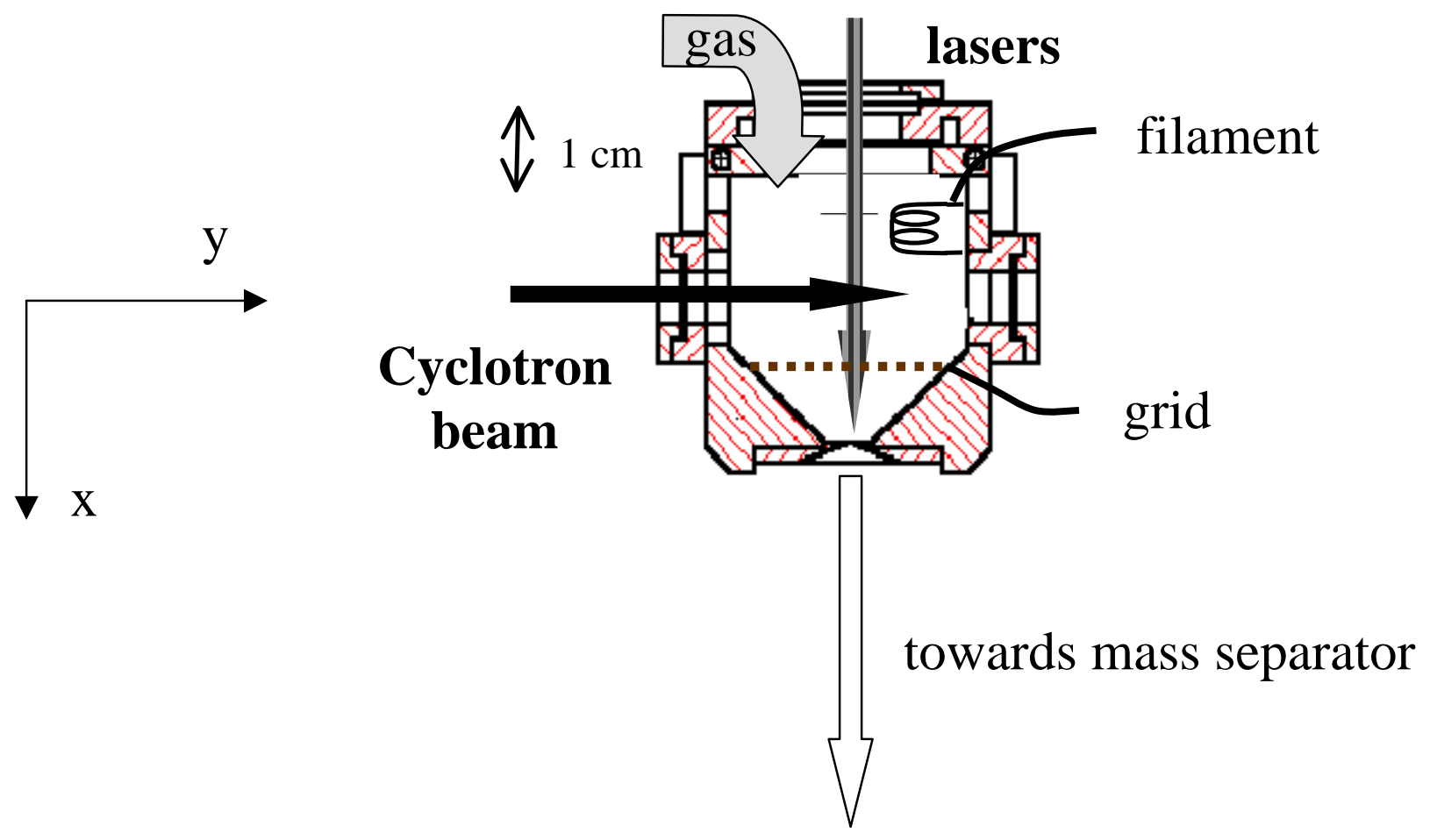

Figure 2 


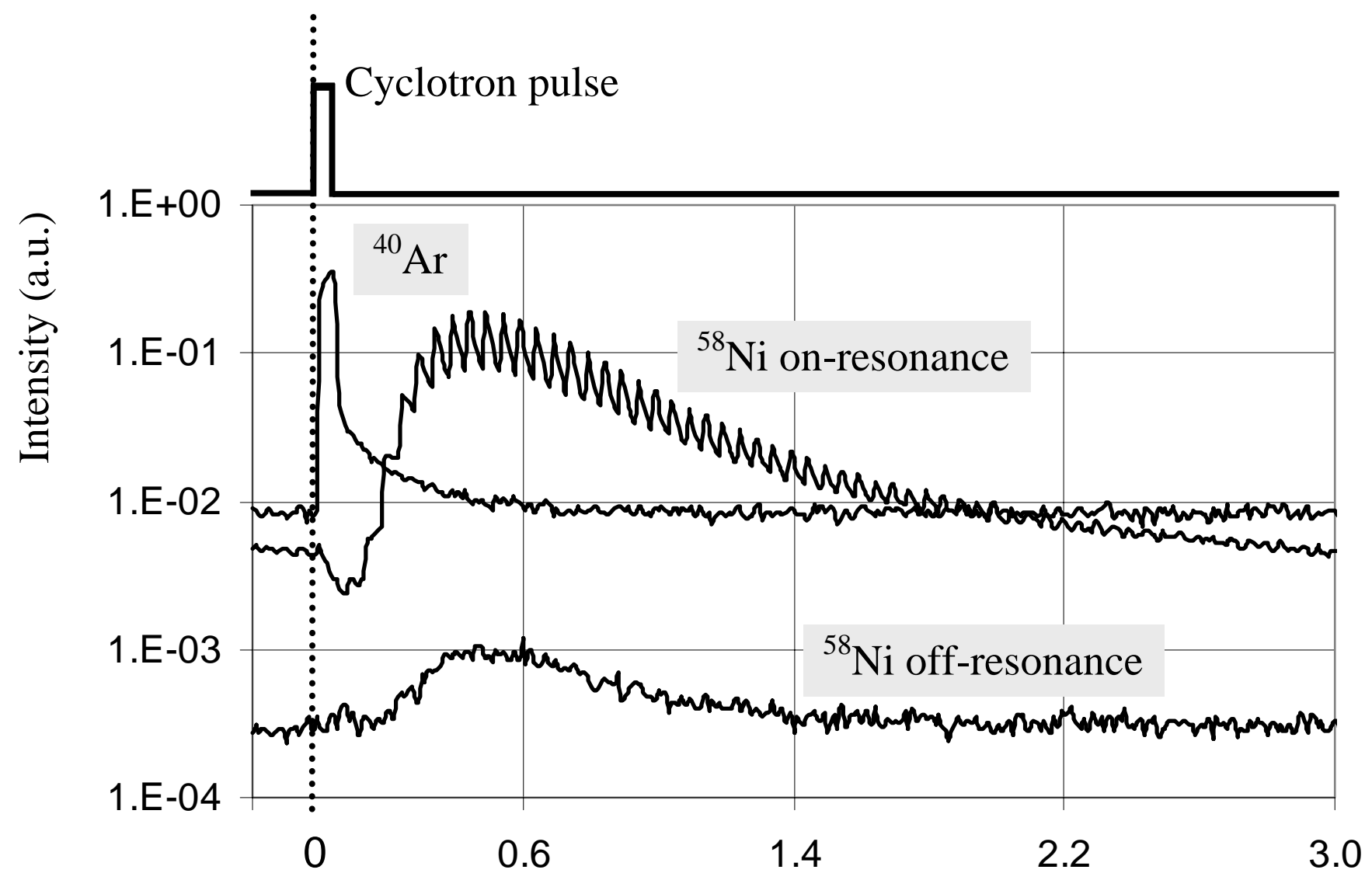

Time (s)

Figure 3 


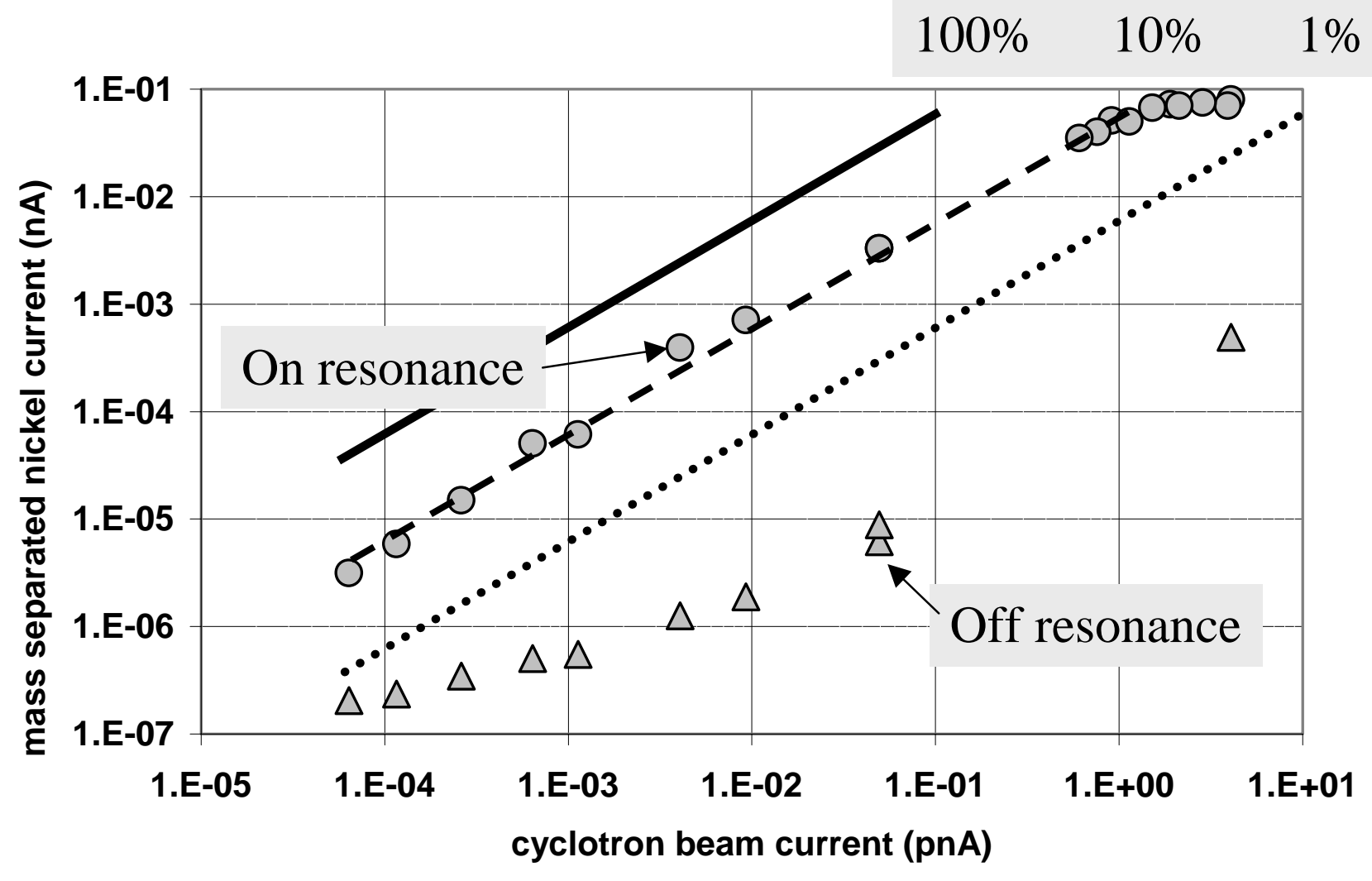

Figure 4 


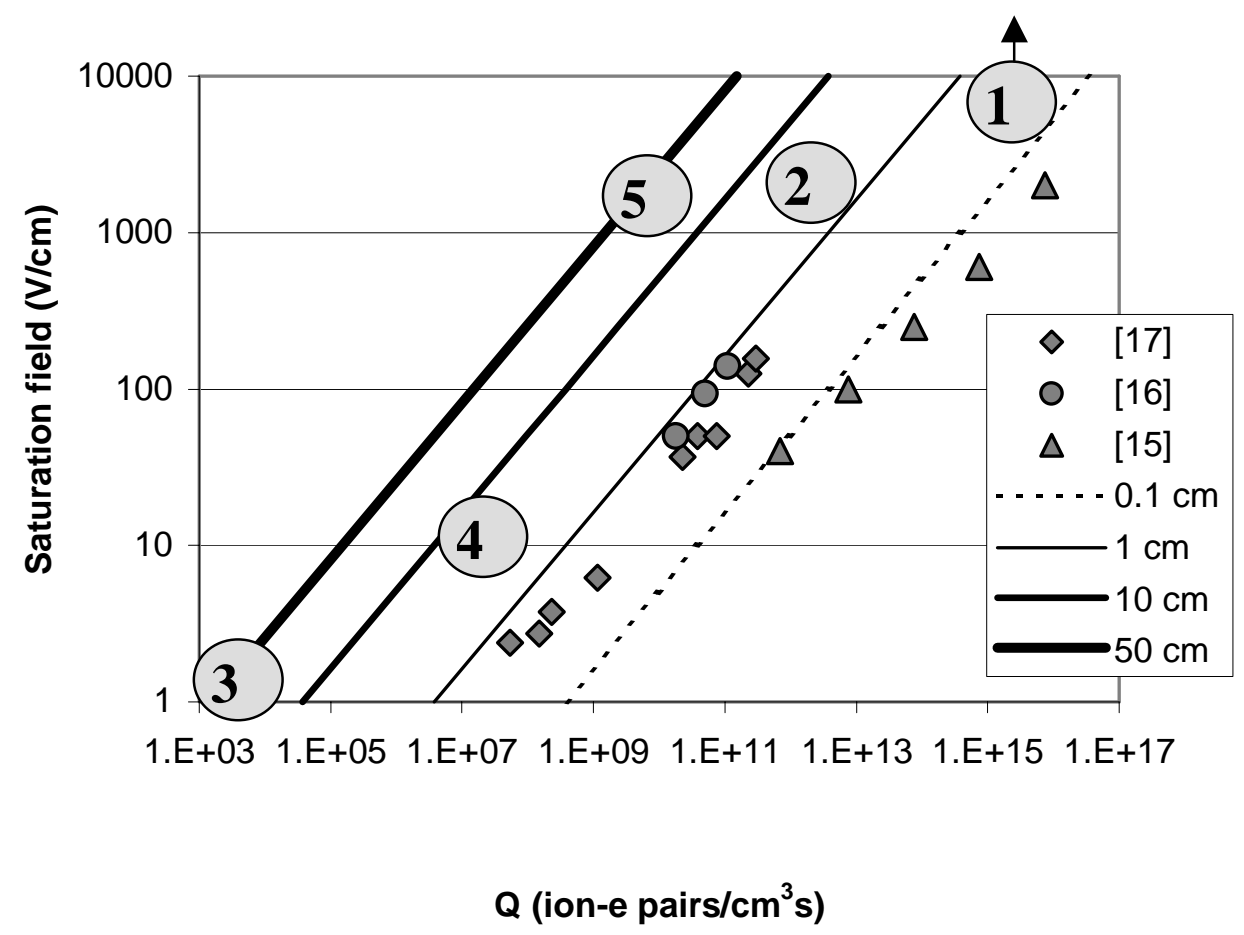

Figure 5 


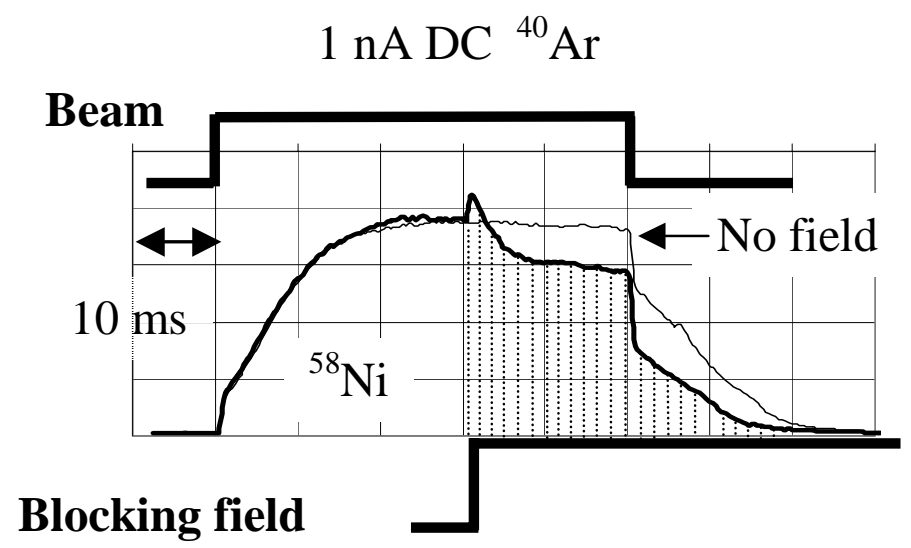

Figure 6 


\begin{tabular}{|c|c|c|c|c|c|}
\hline Gas & $\begin{array}{c}\lambda \\
(\mathrm{cm})\end{array}$ & $\begin{array}{c}\mathrm{v}_{\mathrm{av}} \\
(\mathrm{cm} / \mathrm{s})\end{array}$ & $\begin{array}{c}\mathrm{W}_{\mathrm{i}} \\
(\mathrm{eV})\end{array}$ & $\begin{array}{c}\mathrm{D}^{+} \\
\left(\mathrm{cm}^{2} / \mathrm{s}\right)\end{array}$ & $\begin{array}{c}\mu^{+} \\
\left(\mathrm{cm}^{2} / \mathrm{V} . \mathrm{s}\right)\end{array}$ \\
\hline $\mathrm{H}_{2}$ & $1.8 \times 10^{-5}$ & $2 \times 10^{5}$ & 37 & 0.34 & 13.0 \\
\hline $\mathrm{He}$ & $2.8 \times 10^{-5}$ & $1.4 \times 10^{5}$ & 41 & 0.26 & 10.2 \\
\hline $\mathrm{Ar}$ & $1.0 \times 10^{-5}$ & $4.4 \times 10^{4}$ & 26 & 0.04 & 1.7 \\
\hline $\mathrm{O}_{2}$ & $1.0 \times 10^{-5}$ & $5 \times 10^{4}$ & 31 & 0.06 & 2.2 \\
\hline
\end{tabular}

Table 1

\begin{tabular}{|c|c|c|c|c|c|c|}
\hline $\mathrm{n}_{\mathrm{i}}\left(\mathrm{cm}^{-3}\right)$ & $1 . \mathrm{E}+03$ & $1 . \mathrm{E}+05$ & $1 . \mathrm{E}+07$ & $1 . \mathrm{E}+09$ & $1 . \mathrm{E}+11$ & $1 . \mathrm{E}+13$ \\
\hline $\mathrm{V}_{\text {appl. }} / \mathrm{d}(\mathrm{V} / \mathrm{cm})$ & $1.44 \mathrm{E}-05$ & $3.11 \mathrm{E}-04$ & $6.69 \mathrm{E}-03$ & $1.44 \mathrm{E}-01$ & $3.11 \mathrm{E}+00$ & $6.69 \mathrm{E}+01$ \\
\hline
\end{tabular}

Table 2

\begin{tabular}{|c|c|c|}
\hline $\begin{array}{c}\mathrm{E} \\
(\mathrm{V} / \mathrm{cm})\end{array}$ & $\begin{array}{c}\mathrm{E} / \mathrm{N} \text { (Townsend) } \\
\text { @ } 0.5 \mathrm{~atm}\end{array}$ & $\mathrm{v}_{-}(\mathrm{cm} / \mathrm{s})$ \\
\hline 10 & 0.082 & $1.110^{5}$ \\
\hline 50 & 0.41 & $310^{5}$ \\
\hline 250 & 2.05 & $610^{5}$ \\
\hline 500 & 4.1 & $110^{6}$ \\
\hline 1000 & 8.2 & $1.810^{6}$ \\
\hline
\end{tabular}

Table 3 


\begin{tabular}{|c|c|c|c|c|c|c|c|c|}
\hline & \multicolumn{2}{|l|}{$\mathrm{d}=1 \mathrm{~cm}$} & \multicolumn{2}{|l|}{$d=10 \mathrm{~cm}$} & \multicolumn{2}{|l|}{$\mathrm{d}=50 \mathrm{~cm}$} \\
\hline & & & $1 \%$ loss & $10 \%$ loss & $1 \%$ loss & $10 \%$ loss & $1 \%$ loss & $10 \%$ loss \\
\hline$E$ & $V_{\text {ion }}$ & $\mathrm{V}_{\text {elektron }}$ & $Q$ & $Q$ & $Q$ & $Q$ & $Q$ & $Q$ \\
\hline $\mathrm{V} / \mathrm{cm}$ & $(\mathrm{cm} / \mathrm{s})$ & $(\mathrm{cm} / \mathrm{s})$ & $\left(\mathrm{cm}^{-3} \cdot \mathrm{s}^{-1}\right)$ & $\left(\mathrm{cm}^{-3} \cdot \mathrm{s}^{-1}\right)$ & $\left(\mathrm{cm}^{-3} \cdot \mathrm{s}^{-1}\right)$ & $\left(\mathrm{cm}^{-3} \cdot \mathrm{s}^{-1}\right)$ & $\left(\mathrm{cm}^{-3} \cdot \mathrm{s}^{-1}\right)$ & $\left(\mathrm{cm}^{-3} \cdot \mathrm{s}^{-1}\right)$ \\
\hline 10 & $1.0 \mathrm{E}+02$ & $1.1 \mathrm{E}+05$ & $2.1 \mathrm{E}+12$ & $2.1 \mathrm{E}+13$ & $2.1 \mathrm{E}+10$ & $2.1 \mathrm{E}+11$ & $8.3 E+08$ & $8.3 E+09$ \\
\hline 50 & $5.1 \mathrm{E}+02$ & $3.0 \mathrm{E}+05$ & $2.8 \mathrm{E}+13$ & $2.8 \mathrm{E}+14$ & $2.8 \mathrm{E}+11$ & $2.8 \mathrm{E}+12$ & $1.1 \mathrm{E}+10$ & $1.1 \mathrm{E}+11$ \\
\hline 250 & $2.6 \mathrm{E}+03$ & $6.0 \mathrm{E}+05$ & $2.8 E+14$ & $2.8 E+15$ & $2.8 \mathrm{E}+12$ & $2.8 E+13$ & $1.1 \mathrm{E}+11$ & $1.1 \mathrm{E}+12$ \\
\hline 500 & $5.1 \mathrm{E}+03$ & $1.0 \mathrm{E}+06$ & $9.4 \mathrm{E}+14$ & $9.4 \mathrm{E}+15$ & $9.4 \mathrm{E}+12$ & $9.4 \mathrm{E}+13$ & $3.8 \mathrm{E}+11$ & $3.8 \mathrm{E}+12$ \\
\hline 1000 & $1.0 \mathrm{E}+04$ & $1.8 \mathrm{E}+06$ & $3.4 \mathrm{E}+15$ & $3.4 \mathrm{E}+16$ & $3.4 \mathrm{E}+13$ & $3.4 \mathrm{E}+14$ & $1.4 \mathrm{E}+12$ & $1.4 \mathrm{E}+13$ \\
\hline
\end{tabular}

Table 4.

\begin{tabular}{|c|c|c|c|}
\hline & $\mathrm{d}=1 \mathrm{~cm}$ & $\mathrm{~d}=10 \mathrm{~cm}$ & $\mathrm{~d}=50 \mathrm{~cm}$ \\
\hline E & $\mathrm{Q}\left(\mathrm{V}_{\mathrm{appl}}=\mathrm{V}_{\mathrm{ind}}\right)$ & $\mathrm{Q}\left(\mathrm{V}_{\mathrm{appl}}=\mathrm{V}_{\mathrm{ind}}\right)$ & $\mathrm{Q}\left(\mathrm{V}_{\mathrm{appl}}=\mathrm{V}_{\text {ind }}\right)$ \\
\hline$(\mathrm{V} / \mathrm{cm})$ & (\#ion-e/cm $\left.{ }^{3} . \mathrm{s}\right)$ & (\#ion-e/cm³ ${ }^{3}$.s) & 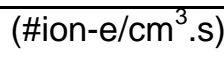 \\
\hline 10 & $2.3 E+09$ & $2.3 E+07$ & $9.0 \mathrm{E}+05$ \\
\hline 50 & $5.6 \mathrm{E}+10$ & $5.6 \mathrm{E}+08$ & $2.3 \mathrm{E}+07$ \\
\hline 100 & $2.3 \mathrm{E}+11$ & $2.3 \mathrm{E}+09$ & $9.0 \mathrm{E}+07$ \\
\hline 250 & $1.4 \mathrm{E}+12$ & $1.4 \mathrm{E}+10$ & $5.6 \mathrm{E}+08$ \\
\hline 500 & $5.6 \mathrm{E}+12$ & $5.6 \mathrm{E}+10$ & $2.3 E+09$ \\
\hline 1000 & $2.3 E+13$ & $2.3 \mathrm{E}+11$ & $9.0 \mathrm{E}+09$ \\
\hline
\end{tabular}

Table 5 


\begin{tabular}{|c|c|c|c|}
\hline case & Incoming beam & gas & $\begin{array}{c}\text { Q } \\
\left(\# \text { ion-e/cm }{ }^{3} . \mathrm{s}\right)\end{array}$ \\
\hline 1 & $1 \mathrm{nA} 200 \mathrm{MeV}^{40} \mathrm{Ar}$ & $0.5 \mathrm{~atm} \mathrm{Ar}$ & $2.6 \times 10^{15}$ \\
\hline 2 & $\begin{array}{c}5.6 \times 10^{8} \text { fission-fragments per s } \\
\text { (induced by } 1 \mu \mathrm{A} 30 \mathrm{MeV} \text { protons) }\end{array}$ & $0.5 \mathrm{~atm} \mathrm{Ar}$ & $5.1 \times 10^{12}$ \\
\hline 3 & $5.5^{242 \mathrm{~m}}$ Am per s (induced by $\left.5 \mu \mathrm{Ad}\right)$ & $0.04 \mathrm{~atm} \mathrm{Ar}$ & $2.6 \times 10^{3}$ \\
\hline 4 & $10^{3} 65 \mathrm{MeV} \mathrm{Po}$ & $0.5 \mathrm{~atm} \mathrm{He}$ & $1.3 \times 10^{7}$ \\
\hline 5 & $10^{8} 650 \mathrm{MeV} \mathrm{Rh}$ & $0.5 \mathrm{~atm} \mathrm{He}$ & $1.6 \times 10^{10}$ \\
\hline
\end{tabular}

Table 6 